\title{
On Dual Hyperbolic Generalized Fibonacci Numbers
}

\author{
Yüksel Soykan \\ Department of Mathematics, Art and Science Faculty, \\ Zonguldak Bülent Ecevit University, 67100, Zonguldak, Turkey \\ e-mail: yuksel_soykan@hotmail.com
}

\begin{abstract}
In this paper, we introduce the generalized dual hyperbolic Fibonacci numbers. As special cases, we deal with dual hyperbolic Fibonacci and dual hyperbolic Lucas numbers. We present Binet's formulas, generating functions and the summation formulas for these numbers. Moreover, we give Catalan's, Cassini's, d'Ocagne's, Gelin-Cesàro's, Melham's identities and present matrices related with these sequences.
\end{abstract}

2010 Mathematics Subject Classification. 11B39, 11B83, 17A45, 05A15.

Keywords. Fibonacci numbers, Lucas numbers, dual hyperbolic numbers, dual hyperbolic Fibonacci numbers, Cassini identity.

\section{Introduction}

The hypercomplex numbers systems, [10], are extensions of real numbers. Some commutative examples of hypercomplex number systems are complex numbers,

$$
\mathbb{C}=\left\{z=a+i b: a, b \in \mathbb{R}, i^{2}=-1\right\},
$$

hyperbolic (double, split-complex) numbers, [12],

$$
\mathbb{H}=\left\{h=a+j b: a, b \in \mathbb{R}, j^{2}=1, j \neq \pm 1\right\},
$$

and dual numbers, [7],

$$
\mathbb{D}=\left\{d=a+\varepsilon b: a, b \in \mathbb{R}, \varepsilon^{2}=0, \varepsilon \neq 0\right\} .
$$

Some non-commutative examples of hypercomplex number systems are quaternions, [8],

$$
\mathbb{H}_{\mathbb{Q}}=\left\{q=a_{0}+i a_{1}+j a_{2}+k a_{3}: a_{0}, a_{1}, a_{2}, a_{3} \in \mathbb{R}, i^{2}=j^{2}=k^{2}=i j k=-1\right\},
$$

octonions [2] and sedenions [13]. The algebras $\mathbb{C}$ (complex numbers), $\mathbb{H}_{\mathbb{Q}}$ (quaternions), $\mathbb{O}$ (octonions) and $\mathbb{S}$ (sedenions) are real algebras obtained from the real numbers $\mathbb{R}$ by a doubling procedure called the CayleyDickson Process. This doubling process can be extended beyond the sedenions to form what are known as the $2^{n}$-ions (see for example [3], [9], [11]). 
Quaternions were invented by Irish mathematician W. R. Hamilton (1805-1865) [8] as an extension to the complex numbers. Hyperbolic numbers with complex coefficients are introduced by J. Cockle in 1848, [6]. H. H. Cheng and S. Thompson [4] introduced dual numbers with complex coefficients and called complex dual numbers. Akar, Yüce and Şahin [1] introduced dual hyperbolic numbers.

A dual hyperbolic number is a hyper-complex number and is defined by

$$
q=\left(a_{0}+j a_{1}\right)+\varepsilon\left(a_{2}+j a_{3}\right)=a_{0}+j a_{1}+\varepsilon a_{2}+\varepsilon j a_{3}
$$

where $a_{0}, a_{1}, a_{2}$ and $a_{3}$ are real numbers.

The set of all dual hyperbolic numbers are denoted by

$$
\mathbb{H}_{\mathbb{D}}=\left\{a_{0}+j a_{1}+\varepsilon a_{2}+\varepsilon j a_{3}: a_{0}, a_{1}, a_{2}, a_{3} \in \mathbb{R}, j^{2}=1, j \neq \pm 1, \varepsilon^{2}=0, \varepsilon \neq 0\right\} .
$$

The base elements $\{1, j, \varepsilon, \varepsilon j\}$ of dual hyperbolic numbers satisfy the following properties (commutative multiplications):

$$
\begin{aligned}
& 1 . \varepsilon=\varepsilon, 1 \cdot j=j, \varepsilon^{2}=\varepsilon \cdot \varepsilon=(j \varepsilon)^{2}=0, j^{2}=j \cdot j=1 \\
& \varepsilon \cdot j=j \cdot \varepsilon, \varepsilon \cdot(\varepsilon j)=(\varepsilon j) \cdot \varepsilon=0, j(\varepsilon j)=(\varepsilon j) j=\varepsilon
\end{aligned}
$$

where $\varepsilon$ denotes the pure dual unit $\left(\varepsilon^{2}=0, \varepsilon \neq 0\right), j$ denotes the hyperbolic unit $\left(j^{2}=1\right)$, and $\varepsilon j$ denotes the dual hyperbolic unit $\left((j \varepsilon)^{2}=0\right)$.

The product of two dual hyperbolic numbers $q=a_{0}+j a_{1}+\varepsilon a_{2}+j \varepsilon a_{3}$ and $p=b_{0}+j b_{1}+\varepsilon b_{2}+j \varepsilon b_{3}$ is

$$
q p=a_{0} b_{0}+a_{1} b_{1}+j\left(a_{0} b_{1}+a_{1} b_{0}\right)+\varepsilon\left(a_{0} b_{2}+a_{2} b_{0}+a_{1} b_{3}+a_{3} b_{1}\right)+j \varepsilon\left(a_{0} b_{3}+a_{1} b_{2}+a_{2} b_{1}+b_{0} a_{3}\right)
$$

and addition of dual hyperbolic numbers is defined as componentwise.

The dual hyperbolic numbers form a commutative ring, real vector space and an algebra. But $\mathbb{H}_{\mathbb{D}}$ is not field because every dual hyperbolic numbers doesn't have an inverse. For more information on the dual hyperbolic numbers, see [1].

Now let us recall the definition of generalized Fibonacci numbers.

A generalized Fibonacci sequence $\left\{V_{n}\right\}_{n \geq 0}=\left\{V_{n}\left(V_{0}, V_{1}\right)\right\}_{n \geq 0}$ is defined by the second-order recurrence relations

$$
V_{n}=V_{n-1}+V_{n-2} ; \quad V_{0}=a, V_{1}=b, \quad(n \geq 2)
$$

with the initial values $V_{0}, V_{1}$ not all being zero. The sequence $\left\{V_{n}\right\}_{n \geq 0}$ can be extended to negative subscripts by defining

$$
V_{-n}=-V_{-(n-1)}+V_{-(n-2)}
$$

for $n=1,2,3, \ldots$ Therefore, recurrence (1.1) holds for all integer $n$.

The first few generalized Fibonacci numbers with positive subscript and negative subscript are given in the following Table 1.

Table 1. A few generalized Fibonacci numbers 


\begin{tabular}{ccc}
\hline$n$ & $V_{n}$ & $V_{-n}$ \\
\hline 0 & $V_{0}$ & \\
1 & $V_{1}$ & $-V_{0}+V_{1}$ \\
2 & $V_{0}+V_{1}$ & $2 V_{0}-V_{1}$ \\
3 & $V_{0}+2 V_{1}$ & $-3 V_{0}+2 V_{1}$ \\
4 & $2 V_{0}+3 V_{1}$ & $5 V_{0}-3 V_{1}$ \\
5 & $3 V_{0}+5 V_{1}$ & $-8 V_{0}+5 V_{1}$
\end{tabular}

If we set $V_{0}=0, V_{1}=1$ then $\left\{V_{n}\right\}$ is the well-known Fibonacci sequence and if we set $V_{0}=2, V_{1}=1$ then $\left\{V_{n}\right\}$ is the well-known Lucas sequence. In other words, Fibonacci sequence $\left\{F_{n}\right\}_{n \geq 0}$ and Lucas sequence $\left\{L_{n}\right\}_{n \geq 0}$ are defined by the second-order recurrence relations

$$
F_{n}=F_{n-1}+F_{n-2}, \quad F_{0}=0, F_{1}=1
$$

and

$$
L_{n}=L_{n-1}+L_{n-2}, \quad L_{0}=2, L_{1}=1 .
$$

The sequences $\left\{F_{n}\right\}_{n \geq 0}$ and $\left\{L_{n}\right\}_{n \geq 0}$ can be extended to negative subscripts by defining

$$
F_{-n}=-F_{-(n-1)}+F_{-(n-2)}
$$

and

$$
L_{-n}=-L_{-(n-1)}+L_{-(n-2)}
$$

for $n=1,2,3, \ldots$ respectively. Therefore, recurrences (1.2) and (1.3) hold for all integer $n$.

We can list some important properties of generalized Fibonacci numbers that are needed.

- Binet formula of generalized Fibonacci sequence can be calculated using its characteristic equation which is given as

$$
t^{2}-t-1=0
$$

The roots of characteristic equation are

$$
\alpha=\frac{1+\sqrt{5}}{2}, \quad \beta=\frac{1-\sqrt{5}}{2} .
$$

Using these roots and the recurrence relation, Binet formula can be given as

$$
V_{n}=\frac{A \alpha^{n}-B \beta^{n}}{\alpha-\beta}
$$

where $A=V_{1}-V_{0} \beta$ and $B=V_{1}-V_{0} \alpha$.

- Binet formula of Fibonacci and Lucas sequences are

$$
F_{n}=\frac{\alpha^{n}-\beta^{n}}{\alpha-\beta}
$$

and

$$
L_{n}=\alpha^{n}+\beta^{n}
$$


respectively.

- The generating function for generalized Fibonacci numbers is

$$
g(t)=\frac{V_{0}+\left(V_{1}-V_{0}\right) t}{1-t-t^{2}}
$$

- The Cassini identity for generalized Fibonacci numbers is

$$
\begin{gathered}
V_{n+1} V_{n-1}-V_{n}^{2}=\left(V_{0} V_{1}-V_{1}^{2}-V_{0}^{2}\right) . \\
A \alpha^{n}=\alpha V_{n}+V_{n-1}, \\
B \beta^{n}=\beta V_{n}+V_{n-1} .
\end{gathered}
$$

In this paper, we define the dual hyperbolic generalized Fibonacci numbers in the next section and give some properties of them.

\section{Dual Hyperbolic Generalized Fibonacci Numbers and their Generating Functions and Binet's Formulas}

In this section, we define dual hyperbolic generalized Fibonacci numbers and present generating functions and Binet formulas for them.

In [5], the authors defined dual hyperbolic Fibonacci and Lucas numbers. We now define dual hyperbolic generalized Fibonacci numbers over $\mathbb{H}_{\mathbb{D}}$. The $n$th dual hyperbolic generalized Fibonacci number is

$$
\widehat{V}_{n}=V_{n}+j V_{n+1}+\varepsilon V_{n+2}+j \varepsilon V_{n+3} .
$$

As special cases, the $n$th dual hyperbolic Fibonacci numbers and the $n$th dual hyperbolic Lucas numbers are given as

$$
\widehat{F}_{n}=F_{n}+j F_{n+1}+\varepsilon F_{n+2}+j \varepsilon F_{n+3}
$$

and

$$
\widehat{L}_{n}=L_{n}+j L_{n+1}+\varepsilon L_{n+2}+j \varepsilon L_{n+3}
$$

respectively. It can be easily shown that

$$
\widehat{V}_{n}=\widehat{V}_{n-1}+\widehat{V}_{n-2}
$$

The sequence $\left\{\widehat{V}_{n}\right\}_{n \geq 0}$ can be extended to negative subscripts by defining

$$
\widehat{V}_{-n}=-\widehat{V}_{-(n-1)}+\widehat{V}_{-(n-2)} .
$$

for $n=1,2,3, \ldots$ respectively. Therefore, recurrence (2.2) holds for all integer $n$.

The first few dual hyperbolic generalized Fibonacci numbers with positive subscript and negative subscript are given in the following Table 2 .

Table 2. A few dual hyperbolic 
generalized Fibonacci numbers

\begin{tabular}{ccc}
\hline$n$ & $\widehat{V}_{n}$ & $\widehat{V}_{-n}$ \\
\hline 0 & $\widehat{V}_{0}$ & \\
1 & $\widehat{V}_{1}$ & $-\widehat{V}_{0}+\widehat{V}_{1}$ \\
2 & $\widehat{V}_{0}+\widehat{V}_{1}$ & $2 \widehat{V}_{0}-\widehat{V}_{1}$ \\
3 & $\widehat{V}_{0}+2 \widehat{V}_{1}$ & $-3 \widehat{V}_{0}+2 \widehat{V}_{1}$ \\
4 & $2 \widehat{V}_{0}+3 \widehat{V}_{1}$ & $5 \widehat{V}_{0}-3 \widehat{V}_{1}$ \\
5 & $3 \widehat{V}_{0}+5 \widehat{V}_{1}$ & $-8 \widehat{V}_{0}+5 \widehat{V}_{1}$
\end{tabular}

Note that

$$
\begin{aligned}
& \widehat{V}_{0}=V_{0}+j V_{1}+\varepsilon V_{2}+j \varepsilon V_{3}=V_{0}+j V_{1}+\varepsilon\left(V_{0}+V_{1}\right)+j \varepsilon\left(V_{0}+2 V_{1}\right), \\
& \widehat{V}_{1}=V_{1}+j V_{2}+\varepsilon V_{3}+j \varepsilon V_{4}=V_{1}+j\left(V_{0}+V_{1}\right)+\varepsilon\left(V_{0}+2 V_{1}\right)+j \varepsilon\left(2 V_{0}+3 V_{1}\right) .
\end{aligned}
$$

For dual hyperbolic Fibonacci numbers (taking $V_{n}=F_{n}, F_{0}=0, F_{1}=1$ ) we get

$$
\begin{aligned}
& \widehat{F}_{0}=j+\varepsilon+2 j \varepsilon, \\
& \widehat{F}_{1}=1+j+2 \varepsilon+3 j \varepsilon,
\end{aligned}
$$

and for dual hyperbolic Lucas numbers (taking $V_{n}=L_{n}, L_{0}=2, L_{1}=1$ ) we get

$$
\begin{aligned}
& \widehat{L}_{0}=2+j+3 \varepsilon+4 j \varepsilon, \\
& \widehat{L}_{1}=1+3 j+4 \varepsilon+7 j \varepsilon .
\end{aligned}
$$

A few dual hyperbolic Fibonacci numbers and dual hyperbolic Lucas numbers with positive subscript and negative subscript are given in the following Table 3 and Table 4.

Table 3. Dual hyperbolic Fibonacci numbers

\begin{tabular}{ccc}
\hline$n$ & $\widehat{F}_{n}$ & $\widehat{F}_{-n}$ \\
\hline 0 & $j+\varepsilon+2 j \varepsilon$ & \\
1 & $1+j+2 \varepsilon+3 j \varepsilon$ & $1+\varepsilon+j \varepsilon$ \\
2 & $1+2 j+3 \varepsilon+5 j \varepsilon$ & $-1+j+j \varepsilon$ \\
3 & $2+3 j+5 \varepsilon+8 j \varepsilon$ & $2-j+\varepsilon$ \\
4 & $3+5 j+8 \varepsilon+13 j \varepsilon$ & $-3+2 j-\varepsilon+j \varepsilon$ \\
5 & $5+8 j+13 \varepsilon+21 j \varepsilon$ & $5-3 j+2 \varepsilon-j \varepsilon$
\end{tabular}

Table 4. Dual hyperbolic Lucas numbers

\begin{tabular}{ccc}
\hline$n$ & $\widehat{L}_{n}$ & $\widehat{L}_{-n}$ \\
\hline 0 & $2+j+3 \varepsilon+4 j \varepsilon$ & \\
1 & $1+3 j+4 \varepsilon+7 j \varepsilon$ & $-1+2 j+\varepsilon+3 j \varepsilon$ \\
2 & $3+4 j+7 \varepsilon+11 j \varepsilon$ & $3+2 \varepsilon-j+j \varepsilon$ \\
3 & $4+7 j+11 \varepsilon+18 j \varepsilon$ & $-4+3 j-\varepsilon+2 j \varepsilon$ \\
4 & $7+11 j+18 \varepsilon+29 j \varepsilon$ & $7+3 \varepsilon-4 j-j \varepsilon$ \\
5 & $11+18 j+29 \varepsilon+47 j \varepsilon$ & $-11+7 j-4 \varepsilon+3 j \varepsilon$ \\
\hline
\end{tabular}

Now, we will state Binet's formula for the dual hyperbolic generalized Fibonacci numbers and in the rest of the paper, we fix the following notations:

$$
\begin{aligned}
& \widehat{\alpha}=1+j \alpha+\varepsilon \alpha^{2}+j \varepsilon \alpha^{3}, \\
& \widehat{\beta}=1+j \beta+\varepsilon \beta^{2}+j \varepsilon \beta^{3} .
\end{aligned}
$$


Note that we have the following identities:

$$
\begin{aligned}
\widehat{\alpha} & =1+j \alpha+\varepsilon(\alpha+1)+j \varepsilon(2 \alpha+1), \\
\widehat{\beta} & =1+j \beta+\varepsilon(\beta+1)+j \varepsilon(2 \beta+1), \\
\widehat{\alpha}^{2} & =\alpha+2+2 \alpha j+2(4 \alpha+3) \varepsilon+4(2 \alpha+1) j \varepsilon, \\
\widehat{\beta}^{2} & =\beta+2+2 \beta j+2(4 \beta+3) \varepsilon+4(2 \beta+1) j \varepsilon, \\
\widehat{\alpha} \widehat{\beta} & =j+3 j \varepsilon, \\
\widehat{\alpha}^{2} \widehat{\beta} & =\alpha+j+(1+5 \alpha) \varepsilon+(4+\alpha) j \varepsilon, \\
\widehat{\alpha} \widehat{\beta}^{2} & =\beta+j+(1+5 \beta) \varepsilon+(4+\beta) j \varepsilon, \\
\widehat{\alpha}^{2} \widehat{\beta}^{2} & =1+6 \varepsilon .
\end{aligned}
$$

THEOREM 1. (Binet's Formula) For any integer n, the nth dual hyperbolic generalized Fibonacci number is

$$
\widehat{V}_{n}=\frac{A \widehat{\alpha} \alpha^{n}-B \widehat{\beta} \beta^{n}}{\alpha-\beta}
$$

Proof. Using Binet's formula

$$
V_{n}=\frac{A \alpha^{n}-B \beta^{n}}{\alpha-\beta}
$$

of the generalized Fibonacci numbers, we obtain

$$
\begin{aligned}
\widehat{V}_{n} & =V_{n}+j V_{n+1}+\varepsilon V_{n+2}+j \varepsilon V_{n+3} \\
& =\frac{A \alpha^{n}-B \beta^{n}}{\alpha-\beta}+j \frac{A \alpha^{n+1}-B \beta^{n+1}}{\alpha-\beta}+\varepsilon \frac{A \alpha^{n+2}-B \beta^{n+2}}{\alpha-\beta}+j \varepsilon \frac{A \alpha^{n+3}-B \beta^{n+3}}{\alpha-\beta} \\
& =\frac{A\left(1+j \alpha+\varepsilon \alpha^{2}+j \varepsilon \alpha^{3}\right) \alpha^{n}-B\left(1+j \beta+\varepsilon \beta^{2}+j \varepsilon \beta^{3}\right) \beta^{n}}{\alpha-\beta} .
\end{aligned}
$$

This proves (2.3).

As special cases, for any integer $n$, the Binet's Formula of $n$th dual hyperbolic Fibonacci number is

$$
\widehat{F}_{n}=\frac{\widehat{\alpha} \alpha^{n}-\widehat{\beta} \beta^{n}}{\alpha-\beta}
$$

and the Binet's Formula of $n$th dual hyperbolic Lucas number is

$$
\widehat{L}_{n}=\widehat{\alpha} \alpha^{n}+\widehat{\beta} \beta^{n} .
$$

Next, we present generating function.

THEOREM 2. The generating function for the dual hyperbolic generalized Fibonacci numbers is

$$
\sum_{n=0}^{\infty} \widehat{V}_{n} x^{n}=\frac{\widehat{V}_{0}+\left(\widehat{V}_{1}-\widehat{V}_{0}\right) x}{1-x-x^{2}} .
$$


Proof. Let

$$
g(x)=\sum_{n=0}^{\infty} \widehat{V}_{n} x^{n}
$$

be generating function of the dual hyperbolic generalized Fibonacci numbers. Then, using the definition of the dual hyperbolic generalized Fibonacci numbers, and substracting $x g(x)$ and $x^{2} g(x)$ from $g(x)$, we obtain (note the shift in the index $n$ in the third line)

$$
\begin{aligned}
\left(1-x-x^{2}\right) g(x) & =\sum_{n=0}^{\infty} \widehat{V}_{n} x^{n}-x \sum_{n=0}^{\infty} \widehat{V}_{n} x^{n}-x^{2} \sum_{n=0}^{\infty} \widehat{V}_{n} x^{n} \\
& =\sum_{n=0}^{\infty} \widehat{V}_{n} x^{n}-\sum_{n=0}^{\infty} \widehat{V}_{n} x^{n+1}-\sum_{n=0}^{\infty} \widehat{V}_{n} x^{n+2} \\
& =\sum_{n=0}^{\infty} \widehat{V}_{n} x^{n}-\sum_{n=1}^{\infty} \widehat{V}_{n-1} x^{n}-\sum_{n=2}^{\infty} \widehat{V}_{n-2} x^{n} \\
& =\left(\widehat{V}_{0}+\widehat{V}_{1} x\right)-\widehat{V}_{0} x+\sum_{n=2}^{\infty}\left(\widehat{V}_{n}-\widehat{V}_{n-1}-\widehat{V}_{n-2}\right) x^{n} \\
& =\left(\widehat{V}_{0}+\widehat{V}_{1} x\right)-\widehat{V}_{0} x=\widehat{V}_{0}+\left(\widehat{V}_{1}-\widehat{V}_{0}\right) x
\end{aligned}
$$

Note that we used the recurrence relation $\widehat{V}_{n}=\widehat{V}_{n-1}+\widehat{V}_{n-2}$. Rearranging above equation, we get

$$
g(x)=\frac{\widehat{V}_{0}+\left(\widehat{V}_{1}-\widehat{V}_{0}\right) x}{1-x-x^{2}} .
$$

As special cases, the generating functions for the dual hyperbolic Fibonacci and dual hyperbolic Lucas numbers are

$$
\sum_{n=0}^{\infty} \widehat{F}_{n} x^{n}=\frac{(j+\varepsilon+2 j \varepsilon)+(1+\varepsilon+j \varepsilon) x}{1-x-x^{2}}
$$

and

$$
\sum_{n=0}^{\infty} \widehat{L}_{n} x^{n}=\frac{(2+j+3 \varepsilon+4 j \varepsilon)+(-1+2 j+\varepsilon+3 j \varepsilon) x}{1-x-x^{2}}
$$

respectively.

\section{Obtaining Binet Formula From Generating Function}

We next find Binet formula of dual hyperbolic generalized Fibonacci number $\left\{\widehat{V}_{n}\right\}$ by the use of generating function for $\widehat{V}_{n}$.

THEOREM 3. (Binet formula of dual hyperbolic generalized Fibonacci numbers)

$$
\widehat{V}_{n}=\frac{d_{1} \alpha^{n}}{(\alpha-\beta)}-\frac{d_{2} \beta^{n}}{(\alpha-\beta)}
$$

where

$$
\begin{aligned}
& d_{1}=\widehat{V}_{0} \alpha+\left(\widehat{V}_{1}-\widehat{V}_{0}\right), \\
& d_{2}=\widehat{V}_{0} \beta+\left(\widehat{V}_{1}-\widehat{V}_{0}\right) .
\end{aligned}
$$


Proof. Let

$$
h(x)=1-x-x^{2}
$$

Then for some $\alpha$ and $\beta$ we write

$$
h(x)=(1-\alpha x)(1-\beta x)
$$

i.e.,

$$
1-x-x^{2}=(1-\alpha x)(1-\beta x)
$$

Hence $\frac{1}{\alpha}$ ve $\frac{1}{\beta}$ are the roots of $h(x)$. This gives $\alpha$ and $\beta$ as the roots of

$$
h\left(\frac{1}{x}\right)=1-\frac{1}{x}-\frac{1}{x^{2}}=0 .
$$

This implies $x^{2}-x-1=0$. Now, by (2.6) and (3.2), it follows that

$$
\sum_{n=0}^{\infty} \widehat{V}_{n} x^{n}=\frac{\widehat{V}_{0}+\left(\widehat{V}_{1}-\widehat{V}_{0}\right) x}{(1-\alpha x)(1-\beta x)}
$$

Then we write

$$
\frac{\widehat{V}_{0}+\left(\widehat{V}_{1}-\widehat{V}_{0}\right) x}{(1-\alpha x)(1-\beta x)}=\frac{A_{1}}{(1-\alpha x)}+\frac{A_{2}}{(1-\beta x)} .
$$

So

$$
\widehat{V}_{0}+\left(\widehat{V}_{1}-\widehat{V}_{0}\right) x=A_{1}(1-\beta x)+A_{2}(1-\alpha x) .
$$

If we consider $x=\frac{1}{\alpha}$, we get $\widehat{V}_{0}+\left(\widehat{V}_{1}-\widehat{V}_{0}\right) \frac{1}{\alpha}=A_{1}\left(1-\beta \frac{1}{\alpha}\right)$. This gives

$$
A_{1}=\frac{\widehat{V}_{0} \alpha+\left(\widehat{V}_{1}-\widehat{V}_{0}\right)}{(\alpha-\beta)}=\frac{d_{1}}{(\alpha-\beta)} \text {. }
$$

Similarly, we obtain

$$
\widehat{V}_{0}+\left(\widehat{V}_{1}-\widehat{V}_{0}\right) \frac{1}{\beta}=A_{2}\left(1-\alpha \frac{1}{\beta}\right) \Rightarrow \widehat{V}_{0} \beta+\left(\widehat{V}_{1}-\widehat{V}_{0}\right)=A_{2}(\beta-\alpha)
$$

and so

$$
A_{2}=-\frac{\widehat{V}_{0} \beta+\left(\widehat{V}_{1}-\widehat{V}_{0}\right)}{(\alpha-\beta)}=-\frac{d_{2}}{(\alpha-\beta)} .
$$

Thus (3.3) can be written as

$$
\sum_{n=0}^{\infty} \widehat{V}_{n} x^{n}=A_{1}(1-\alpha x)^{-1}+A_{2}(1-\beta x)^{-1}
$$

This gives

$$
\sum_{n=0}^{\infty} \widehat{V}_{n} x^{n}=A_{1} \sum_{n=0}^{\infty} \alpha^{n} x^{n}+A_{2} \sum_{n=0}^{\infty} \beta^{n} x^{n}=\sum_{n=0}^{\infty}\left(A_{1} \alpha^{n}+A_{2} \beta^{n}\right) x^{n} .
$$

Therefore, comparing coefficients on both sides of the above equality, we obtain

$$
\widehat{V}_{n}=A_{1} \alpha^{n}+A_{2} \beta^{n}
$$

and then we get (3.1). 
Note that from (2.3) and (3.1) we have

$$
\begin{aligned}
\left(V_{1}-V_{0} \beta\right) \widehat{\alpha} & =\widehat{V}_{0} \alpha+\left(\widehat{V}_{1}-\widehat{V}_{0}\right), \\
\left(V_{1}-V_{0} \alpha\right) \widehat{\beta} & =\widehat{V}_{0} \beta+\left(\widehat{V}_{1}-\widehat{V}_{0}\right) .
\end{aligned}
$$

Next, using Theorem 3, we present the Binet formulas of dual hyperbolic Fibonacci and dual hyperbolic Lucas numbers.

Corollary 4. Binet formulas of dual hyperbolic Fibonacci and dual hyperbolic Lucas numbers are

$$
\widehat{F}_{n}=\frac{\widehat{\alpha} \alpha^{n}-\widehat{\beta} \beta^{n}}{\alpha-\beta}
$$

and

$$
\widehat{L}_{n}=\widehat{\alpha} \alpha^{n}+\widehat{\beta} \beta^{n}
$$

respectively.

\section{Some Identities}

We now present a few special identities for the dual hyperbolic generalized Fibonacci sequence $\left\{\widehat{V}_{n}\right\}$. The following theorem presents the Catalan's identity for the dual hyperbolic generalized Fibonacci numbers.

Theorem 5. (Catalan's identity) For all integers $n$ and $m$, the following identity holds

$$
\widehat{V}_{n+m} \widehat{V}_{n-m}-\widehat{V}_{n}^{2}=\frac{(-1)^{n-m+1}\left((A+B) V_{2 m-1}+(A \beta+B \alpha) V_{2 m}-2(-1)^{m} A B\right)}{5}(j+3 j \varepsilon) .
$$

Proof. Using the Binet Formula

$$
\widehat{V}_{n}=\frac{A \widehat{\alpha} \alpha^{n}-B \widehat{\beta} \beta^{n}}{\alpha-\beta}
$$

and

$$
\begin{aligned}
& A \alpha^{n}=\alpha V_{n}+V_{n-1}, \\
& B \beta^{n}=\beta V_{n}+V_{n-1},
\end{aligned}
$$

we get

$$
\begin{aligned}
\widehat{V}_{n+m} \widehat{V}_{n-m}-\widehat{V}_{n}^{2} & =\frac{\left(A \widehat{\alpha} \alpha^{n+m}-B \widehat{\beta} \beta^{n+m}\right)\left(A \widehat{\alpha} \alpha^{n-m}-B \widehat{\beta} \beta^{n-m}\right)-\left(A \widehat{\alpha} \alpha^{n}-B \widehat{\beta} \beta^{n}\right)^{2}}{(\alpha-\beta)^{2}} \\
& =\frac{-A B \widehat{\alpha} \widehat{\beta} \alpha^{n+m} \beta^{n-m}-A B \widehat{\beta} \widehat{\alpha} \alpha^{n-m} \beta^{n+m}+2 A B \widehat{\alpha} \widehat{\beta} \alpha^{n} \beta^{n}}{(\alpha-\beta)^{2}} \\
& =\frac{-A B \widehat{\alpha} \widehat{\beta} \alpha^{n+m} \beta^{n-m}-A B \widehat{\alpha} \widehat{\beta} \alpha^{n-m} \beta^{n+m}+2 A B \widehat{\alpha} \widehat{\beta} \alpha^{n} \beta^{n}}{(\alpha-\beta)^{2}} \\
& =-A B \widehat{\alpha} \widehat{\beta} \frac{\left(\alpha^{m}-\beta^{m}\right)^{2}}{(\alpha-\beta)^{2}} \alpha^{n-m} \beta^{n-m} \\
& =\frac{(-1)^{n-m+1} A B\left(\alpha^{m}-\beta^{m}\right)^{2}}{5} \widehat{\alpha} \widehat{\beta} \\
& =\frac{(-1)^{n-m+1}\left((A+B) V_{2 m-1}+(A \beta+B \alpha) V_{2 m}-2(-1)^{m} A B\right)}{5}(j+3 j \varepsilon)
\end{aligned}
$$


where $\alpha \beta=-1$ and $\widehat{\alpha} \widehat{\beta}=j+3 j \varepsilon$.

As special cases of the above theorem, we give Catalan's identity of dual hyperbolic Fibonacci and dual hyperbolic Lucas numbers. Firstly, we present Catalan's identity of dual hyperbolic Fibonacci numbers.

Corollary 6. (Catalan's identity for the dual hyperbolic Fibonacci numbers) For all integers $n$ and $m$, the following identity holds

$$
\widehat{F}_{n+m} \widehat{F}_{n-m}-\widehat{F}_{n}^{2}=(-1)^{n-m+1} F_{m}^{2}(j+3 j \varepsilon) .
$$

Proof. Taking $V_{n}=F_{n}$ in Theorem 5 and using the identity

$$
F_{m}^{2}=\frac{2 F_{2 m-1}+F_{2 m}-2(-1)^{m}}{5}
$$

we get the required result.

Secondly, we give Catalan's identity of dual hyperbolic Lucas numbers.

COROLlary 7. (Catalan's identity for the dual hyperbolic Lucas numbers) For all integers $n$ and $m$, the following identity holds

$$
\begin{aligned}
\widehat{L}_{n+m} \widehat{L}_{n-m}-\widehat{L}_{n}^{2} & =(-1)^{n-m}\left(L_{2 m}-2(-1)^{m}\right)(j+3 j \varepsilon) \\
& =(-1)^{n-m}\left(L_{m}^{2}-4(-1)^{m}\right)(j+3 j \varepsilon) .
\end{aligned}
$$

Proof. Taking $V_{n}=L_{n}$ in Theorem 5 and using the identity

$$
L_{m}^{2}=L_{2 m}+2(-1)^{m}
$$

we get the required result.

Note that for $m=1$ in Catalan's identity, we get the Cassini's identity for the dual hyperbolic generalized Fibonacci sequence.

COROLlary 8. (Cassini's identity) For all integers n, the following identity holds

$$
\widehat{V}_{n+1} \widehat{V}_{n-1}-\widehat{V}_{n}^{2}=(-1)^{n} A B(j+3 j \varepsilon) .
$$

As special cases of Cassini's identity, we give Cassini's identity of dual hyperbolic Fibonacci and dual hyperbolic Lucas numbers. Firstly, we present Cassini's identity of dual hyperbolic Fibonacci numbers.

COROllary 9. (Cassini's identity of dual hyperbolic Fibonacci numbers) For all integers n, the following identity holds

$$
\widehat{F}_{n+1} \widehat{F}_{n-1}-\widehat{F}_{n}^{2}=(-1)^{n}(j+3 j \varepsilon) .
$$

Secondly, we give Cassini's identity of dual hyperbolic Lucas numbers.

Corollary 10. (Cassini's identity of dual hyperbolic Lucas numbers) For all integers n, the following identity holds

$$
\widehat{L}_{n+1} \widehat{L}_{n-1}-\widehat{L}_{n}^{2}=5(-1)^{n+1}(j+3 j \varepsilon) .
$$


The d'Ocagne's, Gelin-Cesàro's and Melham' identities can also be obtained by using the Binet Formula of the dual hyperbolic generalized Fibonacci sequence:

$$
\widehat{V}_{n}=\frac{A \widehat{\alpha} \alpha^{n}-B \widehat{\beta} \beta^{n}}{\alpha-\beta} .
$$

The next theorem presents d'Ocagne's, Gelin-Cesàro's and Melham' identities of the dual hyperbolic generalized Fibonacci sequence $\left\{\widehat{V}_{n}\right\}$.

Theorem 11. Let $n$ and $m$ be any integers. Then the following identities are true:

(a): (d'Ocagne's identity)

$$
\widehat{V}_{m+1} \widehat{V}_{n}-\widehat{V}_{m} \widehat{V}_{n+1}=\left(V_{n} V_{m-1}-V_{m} V_{n-1}\right)(j+3 j \varepsilon) .
$$

(b): (Gelin-Cesàro's identity)

$$
\widehat{V}_{n+2} \widehat{V}_{n+1} \widehat{V}_{n-1} \widehat{V}_{n-2}-\widehat{V}_{n}^{4}=-A^{2} B^{2}(1+6 \varepsilon) .
$$

(c): (Melham's identity)

$$
\begin{aligned}
\widehat{V}_{n+1} \widehat{V}_{n+2} \widehat{V}_{n+6}-\widehat{V}_{n+3}^{3} & =(-1)^{n} A B\left(V_{n+1}+j V_{n}+\left(5 V_{n+1}+V_{n}\right) \varepsilon+\left(5 V_{n}+V_{n-1}\right) j \varepsilon\right) \\
& =\frac{(-1)^{n+1} A B\left(-A \widehat{\alpha} \alpha^{n}+B \widehat{\beta} \beta^{n}\right) \widehat{\alpha} \widehat{\beta}}{(\alpha-\beta)} .
\end{aligned}
$$

Proof.

(a): Using (1.7) and (1.8) we obtain

$$
\begin{aligned}
\widehat{V}_{m+1} \widehat{V}_{n}-\widehat{V}_{m} \widehat{V}_{n+1} & =\frac{A B \widehat{\alpha} \widehat{\beta}\left(-\alpha^{m+1} \beta^{n}-\alpha^{n} \beta^{m+1}+\alpha^{m} \beta^{n+1}+\alpha^{n+1} \beta^{m}\right)}{(\alpha-\beta)^{2}} \\
& =\frac{A B\left(\alpha^{n} \beta^{m}-\alpha^{m} \beta^{n}\right)}{(\alpha-\beta)} \widehat{\alpha} \widehat{\beta} \\
& =\frac{\left(\left(\alpha V_{n}+V_{n-1}\right)\left(\beta V_{m}+V_{m-1}\right)-\left(\alpha V_{m}+V_{m-1}\right)\left(\beta V_{n}+V_{n-1}\right)\right)}{(\alpha-\beta)}(j+3 j \varepsilon) \\
& =\left(V_{n} V_{m-1}-V_{m} V_{n-1}\right)(j+3 j \varepsilon) .
\end{aligned}
$$

(b):

$$
\begin{aligned}
\widehat{V}_{n+2} \widehat{V}_{n+1} \widehat{V}_{n-1} \widehat{V}_{n-2}-\widehat{V}_{n}^{4} & =\frac{-A B \widehat{\alpha} \widehat{\beta}(\alpha \beta)^{n}}{(\alpha \beta)^{3}(\alpha-\beta)^{2}} M_{1} \\
& =-A^{2} B^{2} \widehat{\alpha}^{2} \widehat{\beta}^{2}=-A^{2} B^{2}(1+6 \varepsilon)
\end{aligned}
$$

where

$$
\begin{gathered}
M_{1}=\alpha \beta\left(\alpha^{2}+\beta^{2}+3 \alpha \beta\right)\left(A^{2} \widehat{\alpha}^{2} \alpha^{2 n}+B^{2} \widehat{\beta}^{2} \beta^{2 n}\right)-A B \widehat{\alpha} \widehat{\beta}(\alpha \beta)^{n}\left(\alpha^{4}+\beta^{4}+4 \alpha^{2} \beta^{2}+2 \alpha \beta^{3}+2 \alpha^{3} \beta\right) \\
(\alpha-\beta)^{2}=5, \quad \alpha \beta=-1, \quad \alpha+\beta=1, \quad \alpha \beta\left(\alpha^{2}+\beta^{2}+3 \alpha \beta\right)=0
\end{gathered}
$$

and

$$
\alpha^{4}+\beta^{4}+4 \alpha^{2} \beta^{2}+2 \alpha \beta^{3}+2 \alpha^{3} \beta=5 .
$$


(c): Using (1.7), (1.8) and

$$
\begin{aligned}
& \widehat{\alpha}^{2} \widehat{\beta}=\alpha+j+(1+5 \alpha) \varepsilon+(4+\alpha) j \varepsilon, \\
& \widehat{\alpha}^{2}=\beta+j+(1+5 \beta) \varepsilon+(4+\beta) j \varepsilon,
\end{aligned}
$$

we obtain

$$
\begin{aligned}
\widehat{V}_{n+1} \widehat{V}_{n+2} \widehat{V}_{n+6}-\widehat{V}_{n+3}^{3} & =(-1)^{n+1} A B \widehat{\alpha} \widehat{\beta} M_{2} \\
& =\frac{(-1)^{n+1} A B\left(-A \widehat{\alpha} \alpha^{n}+B \widehat{\beta} \beta^{n}\right) \widehat{\alpha} \widehat{\beta}}{(\alpha-\beta)} \\
& =\frac{(-1)^{n+1} A B\left(-\left(\alpha V_{n}+V_{n-1}\right) \widehat{\alpha}^{2} \widehat{\beta}+\left(\beta V_{n}+V_{n-1}\right) \widehat{\alpha} \widehat{\beta}^{2}\right)}{(\alpha-\beta)} \\
& =(-1)^{n} A B\left(V_{n+1}+j V_{n}+\left(5 V_{n+1}+V_{n}\right) \varepsilon+\left(5 V_{n}+V_{n-1}\right) j \varepsilon\right)
\end{aligned}
$$

where

$$
M_{2}=\frac{-A \widehat{\alpha} \alpha^{n-3}\left(2 \alpha^{2}-3 \alpha^{4}+\alpha^{6}-1\right)\left(\alpha^{2}+1\right)^{2}+B \widehat{\beta} \beta^{n-3}\left(2 \beta^{2}-3 \beta^{4}+\beta^{6}-1\right)\left(\beta^{2}+1\right)^{2}}{(\alpha-\beta)^{3}}
$$

and

$$
\alpha^{-3} \frac{\left(2 \alpha^{2}-3 \alpha^{4}+\alpha^{6}-1\right)\left(\alpha^{2}+1\right)^{2}}{(\alpha-\beta)^{3}}=\frac{\left(\beta^{-3}\left(2 \beta^{2}-3 \beta^{4}+\beta^{6}-1\right)\left(\beta^{2}+1\right)^{2}\right)}{(\alpha-\beta)^{3}}=\frac{1}{\alpha-\beta} .
$$

As special cases of the above theorem, we give the d'Ocagne's, Gelin-Cesàro's and Melham' identities of dual hyperbolic Fibonacci and dual hyperbolic Lucas numbers. Firstly, we present the d'Ocagne's, GelinCesàro's and Melham' identities of dual hyperbolic Fibonacci numbers.

Corollary 12. Let $n$ and $m$ be any integers. Then, for the dual hyperbolic Fibonacci numbers, the following identities are true:

(a): (d'Ocagne's identity)

$$
\widehat{F}_{m+1} \widehat{F}_{n}-\widehat{F}_{m} \widehat{F}_{n+1}=(-1)^{m} F_{n-m}(j+3 j \varepsilon) .
$$

(b): (Gelin-Cesàro's identity)

$$
\widehat{F}_{n+2} \widehat{F}_{n+1} \widehat{F}_{n-1} \widehat{F}_{n-2}-\widehat{F}_{n}^{4}=-(1+6 \varepsilon) .
$$

(c): (Melham's identity)

$$
\widehat{F}_{n+1} \widehat{F}_{n+2} \widehat{F}_{n+6}-\widehat{F}_{n+3}^{3}=(-1)^{n}\left(F_{n+1}+j F_{n}+\left(5 F_{n+1}+F_{n}\right) \varepsilon+\left(5 F_{n}+F_{n-1}\right) j \varepsilon\right) .
$$

Secondly, we present the d'Ocagne's, Gelin-Cesàro's and Melham' identities of dual hyperbolic Lucas numbers.

COROLLARY 13. Let $n$ and $m$ be any integers. Then, for the dual hyperbolic Lucas numbers, the following identities are true: 
(a): (d'Ocagne's identity)

$$
\widehat{L}_{m+1} \widehat{L}_{n}-\widehat{L}_{m} \widehat{L}_{n+1}=\left(L_{n} L_{m-1}-L_{m} L_{n-1}\right)(j+3 j \varepsilon) .
$$

(b): (Gelin-Cesàro's identity)

$$
\widehat{L}_{n+2} \widehat{L}_{n+1} \widehat{L}_{n-1} \widehat{L}_{n-2}-\widehat{L}_{n}^{4}=-25(1+6 \varepsilon) .
$$

(c): (Melham's identity)

$$
\widehat{L}_{n+1} \widehat{L}_{n+2} \widehat{L}_{n+6}-\widehat{L}_{n+3}^{3}=5(-1)^{n+1}\left(L_{n+1}+j L_{n}+\left(5 L_{n+1}+L_{n}\right) \varepsilon+\left(5 L_{n}+L_{n-1}\right) j \varepsilon\right) .
$$

\section{Linear Sums}

In this section, we give the summation formulas of the dual hyperbolic generalized Fibonacci numbers with positive and negatif subscripts. Now, we present the summation formulas of the generalized Fibonacci numbers.

Proposition 14. For the generalized Fibonacci numbers, we have the following formulas:

(a): $\sum_{k=0}^{n} V_{k}=V_{n+2}-V_{1}$.

(b): $\sum_{k=0}^{n} V_{2 k}=V_{2 n+1}-V_{1}+V_{0}$.

(c): $\sum_{k=0}^{n} V_{2 k+1}=V_{2 n+2}-V_{2}+V_{1}$.

Proof. For the proof, see Soykan [14].

Next, we present the formulas which give the summation of the dual hyperbolic generalized Fibonacci numbers.

TheOREM 15. For $n \geq 0$, dual hyperbolic generalized Fibonacci numbers have the following formulas:

(a): $\sum_{k=0}^{n} \widehat{V}_{k}=\widehat{V}_{n+2}-\widehat{V}_{1}$.

(b): $\sum_{k=0}^{n} \widehat{V}_{2 k}=\widehat{V}_{2 n+1}-\widehat{V}_{1}+\widehat{V}_{0}$.

(c): $\sum_{k=0}^{n} \widehat{V}_{2 k+1}=\widehat{V}_{2 n+2}-\widehat{V}_{0}$.

Proof.

(a): Note that using Proposition 14 (a) we get

$$
\begin{aligned}
\sum_{k=0}^{n} V_{k} & =V_{n+2}-V_{1}, \\
\sum_{k=0}^{n} V_{k+1} & =V_{n+3}-\left(V_{1}+V_{0}\right), \\
\sum_{k=0}^{n} V_{k+2} & =V_{n+4}-\left(2 V_{1}+V_{0}\right), \\
\sum_{k=0}^{n} V_{k+3} & =V_{n+5}-\left(3 V_{1}+2 V_{0}\right) .
\end{aligned}
$$


Then it follows that

$$
\begin{aligned}
\sum_{k=0}^{n} \widehat{V}_{k} & =\sum_{k=0}^{n} V_{k}+j \sum_{k=0}^{n} V_{k+1}+\varepsilon \sum_{k=0}^{n} V_{k+2}+j \varepsilon \sum_{k=0}^{n} V_{k+3} \\
& =\left(V_{n+2}-V_{1}\right)+j\left(V_{n+3}-\left(V_{1}+V_{0}\right)\right)+\varepsilon\left(V_{n+4}-\left(2 V_{1}+V_{0}\right)\right)+j \varepsilon\left(V_{n+5}-\left(3 V_{1}+2 V_{0}\right)\right) \\
& =\left(V_{n+2}+j V_{n+3}+\varepsilon V_{n+4}+j \varepsilon V_{n+5}\right)-\left(V_{1}+j\left(V_{0}+V_{1}\right)+\varepsilon\left(V_{0}+2 V_{1}\right)+j \varepsilon\left(2 V_{0}+3 V_{1}\right)\right) \\
& =\widehat{V}_{n+2}-\left(V_{1}+j V_{2}+\varepsilon V_{3}+j \varepsilon V_{4}\right) \\
& =\widehat{V}_{n+2}-\widehat{V}_{1} .
\end{aligned}
$$

This proves (a).

(b): Note that using Proposition 14 (b) and (c) we get

$$
\begin{aligned}
\sum_{k=0}^{n} V_{2 k} & =V_{2 n+1}-V_{1}+V_{0}, \\
\sum_{k=0}^{n} V_{2 k+1} & =V_{2 n+2}-V_{0}, \\
\sum_{k=0}^{n} V_{2 k+2} & =V_{2 n+3}-V_{1}, \\
\sum_{k=0}^{n} V_{2 k+3} & =V_{2 n+4}-V_{1}-V_{0} .
\end{aligned}
$$

Then it follows that

$$
\begin{aligned}
\sum_{k=0}^{n} \widehat{V}_{2 k} & =\sum_{k=0}^{n} V_{2 k}+j \sum_{k=0}^{n} V_{2 k+1}+\varepsilon \sum_{k=0}^{n} V_{2 k+2}+j \varepsilon \sum_{k=0}^{n} V_{2 k+3} \\
& =\left(V_{2 n+1}-V_{1}+V_{0}\right)+j\left(V_{2 n+2}-V_{0}\right)+\varepsilon\left(V_{2 n+3}-V_{1}\right)+j \varepsilon\left(V_{2 n+4}-V_{1}-V_{0}\right) \\
& =\left(V_{2 n+1}+j V_{2 n+2}+\varepsilon V_{2 n+3}+j \varepsilon V_{2 n+4}\right)+\left(\left(-V_{1}+V_{0}\right)+j\left(-V_{0}\right)+\varepsilon\left(-V_{1}\right)+j \varepsilon\left(-V_{1}-V_{0}\right)\right) \\
& =\left(V_{2 n+1}+j V_{2 n+2}+\varepsilon V_{2 n+3}+j \varepsilon V_{2 n+4}\right)+\left(\left(V_{0}-V_{1}\right)+j\left(V_{1}-V_{2}\right)+\varepsilon\left(V_{2}-V_{3}\right)+j \varepsilon\left(V_{3}-V_{4}\right)\right) \\
& =\widehat{V}_{2 n+1}-\left(V_{1}+j V_{2}+\varepsilon V_{3}+j \varepsilon V_{4}\right)+\left(V_{0}+j V_{1}+\varepsilon V_{2}+j \varepsilon V_{3}\right) \\
& =\widehat{V}_{2 n+1}-\widehat{V}_{1}+\widehat{V}_{0} .
\end{aligned}
$$

(c): Note that using Proposition 14 (b) and (c) we get

$$
\sum_{k=0}^{n} V_{2 k+4}=V_{2 n+5}-2 V_{1}-V_{0} .
$$


Then it follows that

$$
\begin{aligned}
\sum_{k=0}^{n} \widehat{V}_{2 k+1} & =\sum_{k=0}^{n} V_{2 k+1}+j \sum_{k=0}^{n} V_{2 k+2}+\varepsilon \sum_{k=0}^{n} V_{2 k+3}+j \varepsilon \sum_{k=0}^{n} V_{2 k+4} \\
& =\left(V_{2 n+2}-V_{0}\right)+j\left(V_{2 n+3}-V_{1}\right)+\varepsilon\left(V_{2 n+4}-V_{1}-V_{0}\right)+j \varepsilon\left(V_{2 n+5}-2 V_{1}-V_{0}\right) \\
& =\left(V_{2 n+2}+j V_{2 n+3}+\varepsilon V_{2 n+4}+j \varepsilon V_{2 n+5}\right)+\left(\left(-V_{0}\right)+j\left(-V_{1}\right)+\varepsilon\left(-V_{1}-V_{0}\right)+j \varepsilon\left(-2 V_{1}-V_{0}\right)\right) \\
& =\widehat{V}_{2 n+2}-\left(V_{0}+j V_{1}+\varepsilon V_{2}+j \varepsilon V_{3}\right) \\
& =\widehat{V}_{2 n+2}-\widehat{V}_{0} .
\end{aligned}
$$

As a first special case of the above theorem, we have the following summation formulas for dual hyperbolic Fibonacci numbers:

COROllary 16. For $n \geq 0$, dual hyperbolic Fibonacci numbers have the following properties:

(a): $\sum_{k=0}^{n} \widehat{F}_{k}=\widehat{F}_{n+2}-\widehat{F}_{1}=\widehat{F}_{n+2}-(1+j+2 \varepsilon+3 j \varepsilon)$.

(b): $\sum_{k=0}^{n} \widehat{F}_{2 k}=\widehat{F}_{2 n+1}-\widehat{F}_{1}+\widehat{F}_{0}=\widehat{F}_{2 n+1}-(1+\varepsilon+j \varepsilon)$.

(c): $\sum_{k=0}^{n} \widehat{F}_{2 k+1}=\widehat{F}_{2 n+2}-\widehat{F}_{0}=\widehat{F}_{2 n+2}-(j+\varepsilon+2 j \varepsilon)$.

As a second special case of the above theorem, we have the following summation formulas for dual hyperbolic Lucas numbers:

COROLlary 17. For $n \geq 0$, dual hyperbolic Lucas numbers have the following properties.

(a): $\sum_{k=0}^{n} \widehat{L}_{k}=\widehat{L}_{n+2}-\widehat{L}_{1}=\widehat{L}_{n+2}-(1+3 j+4 \varepsilon+7 j \varepsilon)$.

(b): $\sum_{k=0}^{n} \widehat{L}_{2 k}=\widehat{L}_{2 n+1}-\widehat{L}_{1}+\widehat{L}_{0}=\widehat{L}_{2 n+1}+(1-2 j-\varepsilon-3 j \varepsilon)$.

(c): $\sum_{k=0}^{n} \widehat{L}_{2 k+1}=\widehat{L}_{2 n+2}-\widehat{L}_{0}=\widehat{L}_{2 n+2}-(2+j+3 \varepsilon+4 j \varepsilon)$.

Now, we present the formula which give the summation formulas of the generalized Fibonacci numbers with negative subscripts.

Proposition 18. For $n \geq 1$ we have the following formulas:

(a): $\sum_{k=1}^{n} V_{-k}=-2 V_{-n-1}-V_{-n-2}+V_{1}$.

(b): $\sum_{k=1}^{n} V_{-2 k}=-V_{-2 n-1}+V_{1}-V_{0}$.

(c): $\sum_{k=1}^{n} V_{-2 k+1}=-V_{-2 n}+V_{0}$.

Proof. This is given in Soykan [14].

Next, we present the formulas which give the summation of the dual hyperbolic generalized Fibonacci numbers with negative subscripts.

TheOREM 19. For $n \geq 1$, dual hyperbolic generalized Fibonacci numbers have the following formulas:

(a): $\sum_{k=1}^{n} \widehat{V}_{-k}=-2 \widehat{V}_{-n-1}-\widehat{V}_{-n-2}+\widehat{V}_{1}$.

(b): $\sum_{k=1}^{n} \widehat{V}_{-2 k}=-\widehat{V}_{-2 n-1}+\widehat{V}_{1}-\widehat{V}_{0}$. 
16

YÜKSEL SOYKAN

(c): $\sum_{k=1}^{n} \widehat{V}_{-2 k+1}=-\widehat{V}_{-2 n}+\widehat{V}_{0}$.

Proof. We prove (a). (b) and (c) can be proved similarly. Note that using Proposition 14 (a) we get

$$
\begin{aligned}
\sum_{k=1}^{n} V_{-k} & =-2 V_{-n-1}-V_{-n-2}+V_{1}, \\
\sum_{k=1}^{n} V_{-k+1} & =-2 V_{-n}-V_{-n-1}+V_{1}+V_{0}, \\
\sum_{k=1}^{n} V_{-k+2} & =-2 V_{-n+1}-V_{-n}+2 V_{1}+V_{0}, \\
\sum_{k=1}^{n} V_{-k+3} & =-2 V_{-n+2}-V_{-n+1}+3 V_{1}+2 V_{0} .
\end{aligned}
$$

Then it follows that

$$
\begin{aligned}
\sum_{k=1}^{n} \widehat{V}_{-k}= & \sum_{k=1}^{n} V_{-k}+j \sum_{k=1}^{n} V_{-k+1}+\varepsilon \sum_{k=1}^{n} V_{-k+2}+j \varepsilon \sum_{k=1}^{n} V_{-k+3} \\
= & \left(-2 V_{-n-1}-V_{-n-2}+V_{1}\right)+j\left(-2 V_{-n}-V_{-n-1}+V_{1}+V_{0}\right) \\
& +\varepsilon\left(-2 V_{-n+1}-V_{-n}+2 V_{1}+V_{0}\right)+j \varepsilon\left(-2 V_{-n+2}-V_{-n+1}+3 V_{1}+2 V_{0}\right) \\
= & -2\left(V_{-n-1}+j V_{-n}+\varepsilon V_{-n+1}+j \varepsilon V_{-n+2}\right)-\left(V_{-n-2}+j V_{-n-1}\right. \\
& \left.+\varepsilon V_{-n}+j \varepsilon V_{-n+1}\right)+\left(V_{1}+j\left(V_{1}+V_{0}\right)+\varepsilon\left(2 V_{1}+V_{0}\right)+j \varepsilon\left(3 V_{1}+2 V_{0}\right)\right) \\
= & -2 \widehat{V}_{-n-1}-\widehat{V}_{-n-2}+\left(V_{1}+j V_{2}+\varepsilon V_{3}+j \varepsilon V_{4}\right) \\
= & -2 \widehat{V}_{-n-1}-\widehat{V}_{-n-2}+\widehat{V}_{1} .
\end{aligned}
$$

This proves (a).

As a first special case of the above theorem, we have the following summation formulas for dual hyperbolic Fibonacci numbers:

Corollary 20. For $n \geq 1$, dual hyperbolic Fibonacci numbers have the following properties:

(a): $\sum_{k=1}^{n} \widehat{F}_{-k}=-2 \widehat{F}_{-n-1}-\widehat{F}_{-n-2}+\widehat{F}_{1}=-2 \widehat{F}_{-n-1}-\widehat{F}_{-n-2}+(1+j+2 \varepsilon+3 j \varepsilon)$.

(b): $\sum_{k=1}^{n} \widehat{F}_{-2 k}=-\widehat{F}_{-2 n-1}+\widehat{F}_{1}-\widehat{F}_{0}=-\widehat{F}_{-2 n-1}+(1+\varepsilon+j \varepsilon)$.

(c): $\sum_{k=1}^{n} \widehat{F}_{-2 k+1}=-\widehat{F}_{-2 n}+\widehat{F}_{0}=-\widehat{F}_{-2 n}+(j+\varepsilon+2 j \varepsilon)$.

As a second special case of the above theorem, we have the following summation formulas for dual hyperbolic Lucas numbers:

Corollary 21. For $n \geq 0$, dual hyperbolic Lucas numbers have the following properties.

(a): $\sum_{k=1}^{n} \widehat{L}_{-k}=-2 \widehat{L}_{-n-1}-\widehat{L}_{-n-2}+\widehat{L}_{1}=-2 \widehat{L}_{-n-1}-\widehat{L}_{-n-2}+(1+3 j+4 \varepsilon+7 j \varepsilon)$.

(b): $\sum_{k=1}^{n} \widehat{L}_{-2 k}=-\widehat{L}_{-2 n-1}+\widehat{L}_{1}-\widehat{L}_{0}=-\widehat{L}_{-2 n-1}+(-1+2 j+\varepsilon+3 j \varepsilon)$.

(c): $\sum_{k=1}^{n} \widehat{L}_{-2 k+1}=-\widehat{L}_{-2 n}+\widehat{L}_{0}=-\widehat{L}_{-2 n}+(2+j+3 \varepsilon+4 j \varepsilon)$. 


\section{Matrices related with Dual Hyperbolic Generalized Fibonacci Numbers}

We define the square matrix $B$ of order 2 as:

$$
B=\left(\begin{array}{ll}
1 & 1 \\
1 & 0
\end{array}\right)
$$

such that $\operatorname{det} B=-1$. Induction proof may be used to establish

$$
B^{n}=\left(\begin{array}{cc}
F_{n+1} & F_{n} \\
F_{n} & F_{n-1}
\end{array}\right)
$$

and (the matrix formulation of $V_{n}$ )

$$
\left(\begin{array}{c}
V_{n+1} \\
V_{n}
\end{array}\right)=\left(\begin{array}{ll}
1 & 1 \\
1 & 0
\end{array}\right)^{n}\left(\begin{array}{l}
V_{1} \\
V_{0}
\end{array}\right) .
$$

Now, we define the matrices $B_{V}$ as

$$
B_{V}=\left(\begin{array}{cc}
\widehat{V}_{3} & \widehat{V}_{2} \\
\widehat{V}_{2} & \widehat{V}_{1}
\end{array}\right)
$$

This matrice $B_{V}$ is called dual hyperbolic generalized Fibonacci matrix. As special cases, dual hyperbolic Fibonacci matrix and dual hyperbolic Lucas matrix are

$$
\begin{aligned}
B_{F} & =\left(\begin{array}{cc}
\widehat{F}_{3} & \widehat{F}_{2} \\
\widehat{F}_{2} & \widehat{F}_{1}
\end{array}\right) \text { and } \\
B_{L} & =\left(\begin{array}{ll}
\widehat{L}_{3} & \widehat{L}_{2} \\
\widehat{L}_{2} & \widehat{L}_{1}
\end{array}\right)
\end{aligned}
$$

respectively.

Theorem 22. For $n \geq 0$, the following is valid:

$$
B_{V}\left(\begin{array}{ll}
1 & 1 \\
1 & 0
\end{array}\right)^{n}=\left(\begin{array}{cc}
\widehat{V}_{n+3} & \widehat{V}_{n+2} \\
\widehat{V}_{n+2} & \widehat{V}_{n+1}
\end{array}\right) .
$$

Proof. We prove by mathematical induction on $n$. If $n=0$, then the result is clear. Now, we assume it is true for $n=k$, that is

$$
B_{V} B^{k}=\left(\begin{array}{cc}
\widehat{V}_{k+3} & \widehat{V}_{k+2} \\
\widehat{V}_{k+2} & \widehat{V}_{k+1}
\end{array}\right) .
$$

If we use (2.1), then we have $\widehat{V}_{k+2}=\widehat{V}_{k+1}+\widehat{V}_{k}$. Then, by induction hypothesis, we obtain

$$
\begin{aligned}
B_{V} B^{k+1} & =\left(B_{V} B^{k}\right) B=\left(\begin{array}{ll}
\widehat{V}_{k+3} & \widehat{V}_{k+2} \\
\widehat{V}_{k+2} & \widehat{V}_{k+1}
\end{array}\right)\left(\begin{array}{ll}
1 & 1 \\
1 & 0
\end{array}\right)=\left(\begin{array}{ll}
\widehat{V}_{k+2}+\widehat{V}_{k+3} & \widehat{V}_{k+3} \\
\widehat{V}_{k+1}+\widehat{V}_{k+2} & \widehat{V}_{k+2}
\end{array}\right) \\
& =\left(\begin{array}{cc}
\widehat{V}_{k+4} & \widehat{V}_{k+3} \\
\widehat{V}_{k+3} & \widehat{V}_{k+2}
\end{array}\right) .
\end{aligned}
$$


Thus, (6.3) holds for all non-negative integers $n$.

REMARK 23. The above theorem is true for $n \leq-1$. It can also be proved by induction.

Corollary 24. For all integers $n$, the following holds:

$$
\widehat{V}_{n+2}=\widehat{V}_{2} F_{n+1}+\widehat{V}_{1} F_{n}
$$

Proof. The proof can be seen by the coefficient of the matrix $B_{V}$ and (6.1).

Taking $V_{n}=F_{n}$ and $V_{n}=L_{n}$, respectively, in the above corollary, we obtain the following results.

Corollary 25. For all integers $n$, the followings are true.

(a): $\widehat{F}_{n+2}=\widehat{F}_{2} F_{n+1}+\widehat{F}_{1} F_{n}$.

(b): $\widehat{L}_{n+2}=\widehat{L}_{2} F_{n+1}+\widehat{L}_{1} F_{n}$.

\section{References}

[1] Akar, M., Yüce S., Şahin, Ş., On the Dual Hyperbolic Numbers and the Complex Hyperbolic Numbers, Journal of Computer Science \& Computational Mathematics, 8(1), 1-6, 2018.

[2] Baez, J., The octonions, Bull. Amer. Math. Soc. 39 (2), 145-205, 2002.

[3] Biss, D.K., Dugger, D., and Isaksen, D.C., Large annihilators in Cayley-Dickson algebras, Communication in Algebra, 36 (2), 632-664, 2008.

[4] Cheng, H. H., Thompson, S., Dual Polynomials and Complex Dual Numbers for Analysis of Spatial Mechanisms, Proc. of ASME 24th Biennial Mechanisms Conference, Irvine, CA, August, 19-22, 1996.

[5] Cihan, A., Azak, A. Z., Güngör, M. A., Tosun, M., A Study on Dual Hyperbolic Fibonacci and Lucas Numbers, An. Şt. Univ. Ovidius Constanta, 27(1), 35-48, 2019.

[6] Cockle, J., On a New Imaginary in Algebra, Philosophical magazine, London-Dublin-Edinburgh, 3(34), 37-47, 1849.

[7] Fjelstad, P., G. Gal Sorin, G.G., n-dimensional Hyperbolic Complex Numbers, Advances in Applied Clifford Algebras, 8(1), 47-68, 1998.

[8] Hamilton, W.R., Elements of Quaternions, Chelsea Publishing Company, New York, 1969.

[9] Imaeda, K., Imaeda, M., Sedenions: algebra and analysis, Applied Mathematics and Computation, 115, 77-88, 2000.

[10] Kantor, I., Solodovnikov, A., Hypercomplex Numbers, Springer-Verlag, New York, 1989.

[11] Moreno, G., The zero divisors of the Cayley-Dickson algebras over the real numbers, Bol. Soc. Mat. Mexicana (3)4, 13-28, 1998.

[12] Sobczyk, G., The Hyperbolic Number Plane, The College Mathematics Journal, 26(4), 268-280, 1995.

[13] Soykan, Y. Tribonacci and Tribonacci-Lucas Sedenions. Mathematics 7(1), 74, 2019.

[14] Soykan, Y., On Summing Formulas For Generalized Fibonacci and Gaussian Generalized Fibonacci Numbers, Advances in Research, 20(2), 1-15, 2019. 